\title{
The Impact of Fading and Interference Cancelation On Node Density - Outage Probability Tradeoff in Wireless Networks
}

\author{
Vladimir Mordachev \\ Electromagnetic Compatibility Laboratory \\ Belorussian State University (BSUIR) \\ P.Brovki St. 6, Minsk 220013, Belarus \\ e-mail: emc@ bsuir.by.
}

\author{
Sergey Loyka \\ School of Information Technology and Engineering \\ University of Ottawa \\ Ontario, Canada, K1N 6N5 \\ e-mail: sergey.loyka@ieee.org
}

\begin{abstract}
Outage probability in wireless networks with random Poisson location of the nodes is studied, including the effects of a broad class of fading processes and of various types of interference cancelation. For all scenarios considered, the total (aggregate) interference power is shown to be dominated by that of the nearest interferer (at the low outage region), which is used as a major performance indicator, instead of a traditionally-used aggregate interference power. This simplifies the problem significantly so that explicit closed-form analysis of the outage probability becomes feasible and the effect of various system/network parameters becomes also explicit, including the impacts of complete/partial interference cancelation, of different types of fading and of linear filtering, either alone or in combination with each other. When a given number of strongest interferers are completely canceled, the outage probability scales down exponentially in this number. Three different models of partial cancelation are considered and compared via their outage probabilities, which are obtained in compact closed form. The partial cancelation level required to eliminate the impact of an interferer is quantified. The effect of a broad class of fading processes (including all popular fading models) is included in the analysis in a straightforward way, which can be positive or negative depending on a particular model and propagation/system parameters.
\end{abstract}

\section{INTRODUCTION}

The effect of interference in wireless networks at the physical layer has been studied from several perspectives [1][7]. A typical statistical model of interference in a network includes a model of spatial location of the nodes, a propagation path loss law (which includes the average path loss and, possibly, large-scale (shadowing) and small-scale (multipath) fading) and a threshold-based receiver performance model. The most popular choice for the model of the node spatial distribution is Poisson point process on a plane [1]-[7]. Based on this model and ignoring the effect of fading, Sousa [2] has obtained the characteristic function $(\mathrm{CF})$ of the aggregate (total) interference at the receiver, which can be transformed into a closed-from probability density function (PDF) in some special cases, and, based on it, the error rates for direct sequence (DS) and frequency hopping (FH) systems. For such a model, the distribution of the distance to nearest (or k-th nearest) interferer and, thus, of its interference power can be found in a compact closed form [9]-[11],[13].

While using the LePage series representation, Ilow and Hatzinakos [3][4] have developed a generic technique to obtain the $\mathrm{CF}$ of aggregate interference from a Poisson point process on a plane (2-D) and in a volume (3-D), which can be used to incorporate the effects of Rayleigh and log-normal fading in a straightforward way. Relying on a homogeneous Poisson point process on a plane, Weber et al [5] have characterized the transmission capacity of the network subject to the outage probability constraint via lower and upper bounds. In a recent work, Weber et al [6] use the same approach to characterize the network transmission capacity when the receivers are able to suppress some powerful interferers, and also include the effect of fading (based on the results in [3][4]) and of the transmission strategy [7].

A common feature of all these studies is the use of aggregate interference (either alone or in the form of signalto-interference-plus-noise ratio), and a common lesson is that it is very difficult to deal with: while the $\mathrm{CF}$ of aggregate interference can be obtained in a closed form, the PDF or cumulative distribution function (CDF) are available only in a few special cases. This limits significantly the amount of insight that can be extracted from such a model and, thus, one has to rely on various bounds and approximations, which also complicate the analysis significantly.

A different approach has been adopted in [9]-[11]: the power of the nearest (dominant) interferer is used to evaluate the performance instead of the total interference power. As a result, closed-form analytical performance evaluation becomes straightforward and significant insight can be extracted from such a model, including the scenarios where most powerful interferers are cancelled, either via linear or nonlinear filtering techniques. Further simplification by considering the low outage probability region makes the effect of various system/network parameters explicit and eliminates the need for numerical analysis of the results.

In this paper, we rely on the approach developed in [9][11] and, using the methods of functions of regular variations, 
show that the aggregate (total) interference is dominated by the nearest interferer in the region of low outage probability (i.e. the practically-important region; see Theorem 1,2 and 3 ) and, thus, both models give the same results, including the scenarios with partial/complete interference cancellation and when fading (form a broad class of distributions) is present.

We consider 3 different techniques of partial cancellation and compare them using compact, closed-form characterization of the outage probabilities and, unlike [6], without any simplifying assumptions. The level of cancelation required to eliminate the impact of an interferer is also quantified. Proper resource allocation is shown to significantly relax this requirement.

The proposed method is further used to include the impact of fading. Specifically, we demonstrate (directly in terms of the outage probability, without using the characteristic function) that the effect of a broad class of fading distributions, which includes all popular models (Rayleigh, Rice, Nakagami, lognormal, composite Rayleigh-log-normal (Suzuki), Weibull etc. [8]; an explicit condition for distributions to belong to this class is given) is a multiplicative constant shift of the outage probability when compared to the no-fading case. In the case of Rayleigh fading, this is a moderate constant (close to 1), and the effect of fading can be either positive (constant $<1$ ) or negative (constant $>1$ ), depending on the path loss exponent and other parameters. In the case of log-normal fading, the constant can be significantly greater than 1 and the effect of fading is always negative. The composite Rayleigh-log-normal fading results in a shift equal to the product of individual shift constants.

We further show that, for all fading distributions considered above, the total interference power is still dominated by the nearest interferer and the typical outage events (total interference power exceeding a threshold) are due to such an interferer being not in a deep fade. Thus, the outage probabilities defined in terms of the total and nearest interferer's power are the same at low outage region. The combined effect of fading and complete/partial interference cancellation is also considered and the main conclusions above are shown to hold in this case as well. It is shown that fading relaxes the requirement to the interference cancellation level.

Comparison of linear filtering to complete/partial cancellation of nearest interferers shows that the complete cancellation (or partial cancellation with the sufficient cancellation level) is most efficient, and that linear filtering and partial cancellation are similar in their impact on the outage probability.

As a by-product of our analysis, some known results (e.g. the effect of fading) originally obtained via elaborate analysis are derived in a straightforward and simple way.

\section{Network AND System Model}

As an interference model of wireless network at the physical layer, we consider a number of point-like transmitters (Tx) and receivers $(\mathrm{Rx})$ that are randomly located over a certain limited region of space $S_{m}$, which can be one $(m=1)$, two $(m=2)$, or three $(m=3)$-dimensional (1-D, 2$\mathrm{D}$ or 3-D). This can model location of the nodes over a highway or a street canyon (1-D), a residential area (2-D), or a downtown area with a number of high-rise buildings (3D). In our analysis, we consider a single (randomly-chosen) receiver and a number of transmitters that generate interference to this receiver, and which follow the Poisson distribution: the probability of exactly $k$ transmitters falling into the region $S$ is given by,

$$
\mathcal{P}(k, S)=e^{-\bar{N}} \bar{N}^{k} / k !
$$

where $\bar{N}=\int_{S} \rho d S$ is the average number of transmitters falling into the region $S$, and $\rho$ is the average node density. If the density is constant, then $\bar{N}=\rho S$. The numbers of nodes in two disconnected areas are statistically independent.

Possible scenarios to which the assumptions above apply, with a certain degree of approximation, are a sensor network with randomly-located non-cooperating sensors; a network(s) of mobile phones from the same or different providers (in the same area); a network of multi-standard wireless devices sharing the same resources (e.g. common or adjacent bands of frequencies) or an ad-hoc network.

Consider now a given transmitter-receiver pair. The power at the Rx antenna output $P_{r}$ coming from the transmitter is given by the standard link budget equation [8]: $P_{r}=P_{t} G_{t} G_{r} g$, where $P_{t}$ is the Tx power, $G_{t}, G_{r}$ are the Tx and Rx antenna gains, and $g$ is the propagation path gain ( $=1 /$ path loss), $g=g_{a} g_{l} g_{s}$, where $g_{a}$ is the average propagation path gain, and $g_{l}, g_{s}$ are the contributions of large-scale (shadowing) and small-scale (multipath) fading, which can be modeled as independent log-normal and Rayleigh (Rice) random variables, respectively [8]. The widely-accepted model for $g_{a}$, which is used here, is $g_{a}=a_{\nu} R^{-\nu}$, where $\nu$ is the path loss exponent, and $a_{\nu}$ is a constant independent of the Tx-Rx distance $R$ [8].

\section{INTERFERENCE TO NOISE RATIO}

In this section, we briefly review the relevant results from [9]-[11]. Let us consider a fixed-position receiver (e.g. a base station of a given user) and a number of randomly located interfering transmitters (interferers, e.g. mobile units of other users) of the same power $P_{t}$. In this section, we ignoring the effect of fading (i.e. $g_{l}=g_{s}=1$; the fading is taken into account in section V), and assume, for simplicity, that the antennas are isotropic (the effect of directional antennas was considered in [11]), and consider the interfering signals at the receiver input.

The statistics of transmitters' location is given by (1). Transmitter $i$ produces the average power $P_{a i}=P_{t} g_{a}\left(R_{i}\right)$ at the receiver input, and we consider only the signals exceeding the Rx noise level $P_{0}, P_{a i} \geq P_{0}$. We define the interference-tonoise ratio (INR) $d_{a}$ (also known as dynamic range [9]-[11]) in the ensemble of the interfering signals via the most powerful 
(at the Rx input) signal ${ }^{1}$,

$$
d_{a}=P_{a 1} / P_{0}
$$

where, without loss of generality, we index the transmitters in the order of decreasing $\mathrm{Rx}$ power, $P_{a 1} \geq P_{a 2} \geq \ldots \geq$ $P_{a N}$. The most powerful signal is coming from the transmitter located at the minimum distance $r_{1}, P_{a 1}=P_{t} g_{a}\left(r_{1}\right)$.

The probability that the INR exceeds value $D$ is $\operatorname{Pr}\left\{d_{a}>D\right\}=\operatorname{Pr}\left\{r_{1}<r(D)\right\}$, where the distance $r(D)$ is such that $P_{a}(r(D))=P_{0} D$, so that the CDF of $d_{a}$ is [9]-[11]

$$
F_{d}(D)=1-\operatorname{Pr}\left\{d_{a}>D\right\}=\exp (-\bar{N}(D))
$$

where $\bar{N}(D)=\int_{V(r(D))} \rho d V$ is the average number of transmitters in the ball $V(r(D))$ of the radius $r(D)=$ $\left(P_{t} a_{\nu} / P_{0} D\right)^{1 / \nu}$.

When the average spatial density of transmitters is constant, $\rho=$ const, (3) simplifies to $F_{d}(D)=\exp \left\{-\bar{N}_{\max } / D^{m / \nu}\right\}$ [9]-[11], where $c_{1}=2, c_{2}=\pi$ and $c_{3}=4 \pi / 3, \bar{N}_{\max }=$ $c_{m} R_{\max }^{m} \rho$ is the average number of transmitters in the ball of radius $R_{\max }$, which we term "potential interference zone", and $R_{\max }$ is such that $P_{a}\left(R_{\max }\right)=P_{0}$, i.e. a transmitter at the boundary of the potential interference zone produces signal at the receiver exactly at the noise level; transmitters located outside of this zone produce weaker signals, which are neglected in the analysis. Note that $F_{d}(D)$ above gives the distribution of the INR as a simple explicit function of the system and geometrical parameters, and ultimately depends on $\bar{N}_{\max }, m, \nu$ only.

When $(k-1)$ most powerful signals, which are coming from $(k-1)$ closest transmitters, do not create any interference (i.e. due to frequency, time or code separation in the multiple access scheme, or due to any other form of separation or filtering), the CDF and PDF of the distance $r_{k}$ to the most powerful interfering signal of order $k$ can be found in a similar way. The CDF of the INR $d_{a}$ in this case is given by

$$
F_{d k}(D)=e^{-\bar{N}(D)} \sum_{i=0}^{k-1} \bar{N}(D)^{i} / i !
$$

In the case of constant average density $\rho=$ const, the CDF and PDF of the INR simplify to [11]

$$
F_{d k}(D)=\exp \left\{-\frac{\bar{N}_{\max }}{D^{m / \nu}}\right\} \sum_{i=0}^{k-1} \frac{1}{i !}\left(\frac{\bar{N}_{\max }}{D^{m / \nu}}\right)^{i} .
$$

\section{Outage Probability-Node Density TradeofF}

Powerful interfering signals can result in significant performance degradation due to linear and nonlinear distortion effects in the receiver when they exceed certain limit, which we characterize here via the receiver distortion-free dynamic range, i.e. the maximum acceptable interference-to-nose ratio, $D_{d f}=P_{\max } / P_{0}$, where $P_{\max }$ is the maximum interference power at the receiver that does not cause significant performance degradation. If $d_{a}>D_{d f}$, there is significant

\footnotetext{
${ }^{1}$ In the next sections, we shown that that, in the small outage region, the total interference power (i.e. coming from all transmitters) is dominated by the contribution of the nearest interferer
}

performance degradation and the receiver is considered to be in outage, which corresponds to one or more transmitters falling into the active interference zone (i.e. the ball of radius $r\left(D_{d f}\right)$; the signal power coming from transmitters at that zone exceeds $\left.P_{\max }\right)$, whose probability is

$$
\mathcal{P}_{\text {out }}=\operatorname{Pr}\left\{d_{a}>D_{d f}\right\}=1-F_{d}\left(D_{d f}\right)
$$

For given $\mathcal{P}_{\text {out }}$, one can find the required distortion-free dynamic range ("outage dynamic range") $D_{d f}$

$$
D_{d f}=F_{d}^{-1}\left(1-\mathcal{P}_{\text {out }}\right)
$$

We note that, in general, $D_{d f}$ is a decreasing function of $\mathcal{P}_{\text {out }}$, i.e. low outage probability calls for high distortion-free dynamic range. For simplicity of notations, we further drop the subscript and denote the distortion-free dynamic range by $D$.

While the definition of outage probability above relies on the maximum interfering power, the same outage probability holds in terms of the total interfering power at the low outage region, as the theorem below demonstrates.

Theorem 1: Consider the outage probability in (6). At the low outage region, it converges to the outage probability defined via the total interference power, i.e.

$$
\lim _{x \rightarrow \infty} \frac{\operatorname{Pr}\left\{\sum_{i} P_{a i}>x\right\}}{\operatorname{Pr}\left\{P_{a 1}>x\right\}}=1
$$

Proof: via the functions of regular variation and using Lemma 4.4.2 in [12].

Thus, at the low outage region, $\mathcal{P}_{\text {out }}$ in (6) serves as an accurate approximation of the outage probability in terms of the total interference power,

$$
\operatorname{Pr}\left\{\sum_{i} P_{a i}>x\right\} \approx \operatorname{Pr}\left\{P_{a 1}>x\right\}, \text { for large } x
$$

and all our results also apply to such an outage probability. A significant advantage of (6) is that a closed-form analysis becomes straightforward.

\section{A. $(k-1)$ nearest interferers are cancelled}

This case has been considered in [11] in terms of the nearest interferer power, which is summarized below (the case of no interference cancellation corresponds to $k=1$ ). We also show, in Theorem 2 below, that the corresponding results hold in terms of the total interference power as well.

Assume that $(k-1)$ nearest interferers are eliminated via some means (e.g. by processing at the receiver or resource allocation). In this case, (4), (5) apply and the outage probability, at low outage region $\left(\mathcal{P}_{\text {out }, 1} \ll 1\right)$, is

$$
\mathcal{P}_{\text {out }} \approx \frac{1}{k !} \bar{N}^{k}=\frac{1}{k !}\left(\frac{\bar{N}_{\max }}{D^{m / \nu}}\right)^{k}
$$

which can be expressed as $\mathcal{P}_{\text {out }}=\frac{1}{k !} \mathcal{P}_{\text {out }, 1}^{k} \leq \mathcal{P}_{\text {out }, 1}$, where $\mathcal{P}_{\text {out }, 1}$ is the outage probability for $k=1$ (no interference cancellation). In the small outage region, $\mathcal{P}_{\text {out }, 1} \ll 1$ and $\mathcal{P}_{\text {out }} \ll \mathcal{P}_{\text {out }, 1}$, i.e. there is a significant beneficial effect of 
removing $(k-1)$ strongest interferers, which scales exponentially with $k$.

In a similar way, the node density-outage probability tradeoff can be formulated. In the small outage probability region $\mathcal{P}_{\text {out }} \ll 1$, it can be expressed as

$$
\bar{N}=\int_{V(r(D))} \rho d V \leq\left(k ! \mathcal{P}_{\text {out }}\right)^{1 / k}
$$

From (11), one can clearly see the beneficial effect of "removing" $(k-1)$ most powerful interferers on the outage probability-network density tradeoff: since $\left(k ! \mathcal{P}_{\text {out }}\right)^{1 / k} \gg$ $\mathcal{P}_{\text {out }}$ in the small outage regime, significantly higher node density is allowed, at the same outage probability, for higher $k$.

In the case of uniform density, (11) reduces to

$$
\rho \leq c_{m}^{-1}\left(k ! \mathcal{P}_{\text {out }}\right)^{1 / k}\left(P_{\max } / P_{t} a_{\nu}\right)^{m / \nu}
$$

Note that the upper bound on the node density scales as $\left(k ! \mathcal{P}_{\text {out }}\right)^{1 / k}$.

These results also hold, in the small outage region, in terms of the total interference power (used to define the outage probability):

Theorem 2: Consider the outage probability in (10). At the low outage region, it converges to the outage probability defined via the total interference power, i.e.

$$
\lim _{x \rightarrow \infty} \frac{\operatorname{Pr}\left\{\sum_{i=k}^{N} P_{a i}>x\right\}}{\operatorname{Pr}\left\{P_{a k}>x\right\}}=1
$$

and, thus, the following approximation holds,

$$
\operatorname{Pr}\left\{\sum_{i=k}^{N} P_{a i}>x\right\} \approx \operatorname{Pr}\left\{P_{a k}>x\right\} \text {, for large } x .
$$

Proof: along the same lines as that of Theorem 1.

\section{B. Partial cancellation of $(k-1)$ nearest interferers}

In this section, we generalize the results in [11] to the case of non-ideal (realistic) interference cancellation, when $(k-1)$ nearest interferers are attenuated by a factor of $0 \leq \alpha \leq 1$ (so that the interference power is $\alpha P_{a i}, 1 \leq i \leq k-1$ ) where $\alpha=0$ corresponds to the ideal case (complete cancellation) and $\alpha=1$ corresponds to the case of no cancellation at all. When $\alpha$ is independent of $D$, it is straightforward to show that asymptotically $(D \rightarrow \infty)$ the nearest interferer dominates the outage probability, which is given by

$$
\mathcal{P}_{\text {out }} \approx \alpha^{m / \nu} \bar{N}_{\max } D^{-m / \nu}, \alpha>0
$$

and which is also the same as that of cancelling only the nearest interferer $(k=2)$, i.e. partial cancelling of more than 1 nearest interferer by a fixed level does not bring any additional advantage asymptotically, and the outage probability in this case significantly exceeds that of complete cancellation (compare (15) to (10)). Comparing (15) to (10) with $k=1$, the effect of partial cancellation by a factor of $\alpha$ is to reduce $\mathcal{P}_{\text {out }}$ by a factor of $\alpha^{m / \nu}$ compared to the no cancellation case, i.e. by a factor of $\alpha$ for $m=2$ and $\nu=2$ (free space propagation) and by a factor of $\sqrt{\alpha}$ for $m=2$ and $\nu=4$ (two-ray propagation or ground reflection).

One can also consider another scenario, where $(k-2)$ nearest interferers are cancelled completely (for example, by proper resource allocation, frequency or time) and $(k-1)$ th interferer is cancelled partially (e.g. by processing at the receiver), $k \geq 3$. In such a case, it is straightforward to show that the $(k-1)$-th interferer dominates asymptotically and the outage probability is given by

$$
\mathcal{P}_{\text {out }} \approx \frac{\alpha^{(k-1) m / \nu}}{(k-1) !}\left(\frac{\bar{N}_{\max }}{D^{m / \nu}}\right)^{k-1}, \alpha>0
$$

i.e. a significant improvement over (15), but still higher than (10) (complete cancellation).

Finally, one can also consider the case where $\alpha$ scales as a function of $D$ and ask a question: "What level of cancellation is required to eliminate the effect of $(k-1)$-th nearest interferer?" Assuming that $(k-2)$ nearest interferers are cancelled completely and comparing the contribution of the partially-cancelled $(k-1)$-th interferer (see $(16))$ to $k$-th interferer (not cancelled at all, see (10)), it is straightforward to show that the $k$-th interferer dominates if

$$
\alpha<\frac{1}{D^{1 /(k-1)}}\left(\frac{\bar{N}_{\max }}{k}\right)^{\nu / m(k-1)}
$$

Thus, perfect cancellation is not a prerequisite and $\alpha>0$ can also do the job, if it properly scales with $D$.

Similar condition can also be obtained when $(k-1)$ nearest interferers are partially cancelled by the same factor $\alpha$,

$$
\alpha<\frac{1}{D^{k-1}}\left(\frac{\bar{N}_{\max }^{k-1}}{k !}\right)^{\nu / m}
$$

which is, however, a significantly tighter requirement than (17), as intuitively expected. Thus, complete cancellation of some nearest interferers (e.g. via resource allocation) is of significant help when only partial (realistic) cancellation at the receiver is possible.

\section{IMPACT OF FADING}

While the effect of fading on the total interference power under the Poisson spatial distribution of interferers has been analysed in [4][5] (using the LePage series representation) in terms of the characteristic function only, our analysis here is explicit in terms of the outage probability and thus provides additional insight into interference-generating mechanisms and their impact. In particular, we demonstrate that the total interference power is dominated by that of the nearest interferer for a broad class of fading distributions (including all popular models), which allows us to derive compact closed-form expressions for the outage probability and also to generalize the results in [11] to the case of fading channel (from a broad class of distributions). 


\section{A. Impact of Rayleigh fading}

Let us consider the ordered average powers $P_{a 1} \geq P_{a 2} \geq$ $\ldots \geq P_{a N}$ which are further subjected to Rayleigh fading so that the fading received powers are $P_{s i}=g_{s i} P_{a i}$, where $g_{s i}$ are the Rayleigh fading factors, assumed to be i.i.d., with the standard pdf $f_{g s}(x)=e^{-x}$. The dynamic range is now defined as $d_{s}=P_{s 1} / P_{0}=d_{a} g_{s 1}$, where $d_{a}=P_{a 1} / P_{0}$, i.e. via the contribution of the nearest interferer ${ }^{2}$, and its cumulative CDF (CCDF), i.e. the outage probability, is

$$
\mathcal{P}_{\text {out }}=\operatorname{Pr}\left\{d_{s}>D\right\}=\int_{0}^{\infty} f_{g s}(g) \bar{F}_{d}(D / g) d g,
$$

where $\bar{F}_{d}(x)=1-F_{d}(x)$ is the CCDF of $d_{a}$. At the low outage region, i.e. at the distribution tail $D \rightarrow \infty$, it can be approximated, after some manipulations, as

$$
\mathcal{P}_{\text {out }} \approx \Gamma(m / \nu+1) \bar{N}_{\max } D^{-m / \nu},
$$

where $\Gamma$ is the gamma function. Comparing to (10) with $k=1$, we conclude that the effect of Rayleigh fading is the multiplicative shift by a constant factor $\Gamma(m / \nu+1)$, and the functional form of the distribution (i.e. regular variation or heavy tail) is preserved. Since $\Gamma(m / \nu+1)$ can be greater or smaller than 1 , depending on $m / \nu$ (e.g. $m=2, \nu=4 \rightarrow \Gamma \approx$ 0.89 ), the effect of Rayleigh fading can be both positive and negative.

In a similar way, one obtains the outage probability when $(k-1)$ nearest interferers are cancelled,

$$
\mathcal{P}_{\text {out }} \approx \frac{\Gamma(k m / \nu+1)}{k !}\left(\frac{\bar{N}_{\max }}{D^{m / \nu}}\right)^{k}
$$

i.e. the beneficial effect of cancelling is slightly offset by fading (since $\Gamma(\mathrm{km} / \nu+1)$ is an increasing function of $k$ ) but otherwise follows the same tendency as without fading (see (10)).

Since the INR is the scaled interference power, the later will follow the same distribution as in (21) (up to a constant) and, thus, $\operatorname{Pr}\left\{P_{s k}>x\right\}$ is a function of regular variation so that Theorem 2 applies, i.e.

Theorem 3: When the interferers are subject to the average path loss and Rayleigh fading, the nearest interferer dominates in terms of the outage probability at the low outage region, i.e.

$$
\begin{gathered}
\lim _{x \rightarrow \infty} \frac{\operatorname{Pr}\left\{\sum_{i=k}^{N} P_{s i}>x\right\}}{\operatorname{Pr}\left\{P_{s k}>x\right\}}=1 \\
\operatorname{Pr}\left\{\sum_{i=k}^{N} P_{s i}>x\right\} \approx \operatorname{Pr}\left\{P_{s k}>x\right\}, \text { for large } x
\end{gathered}
$$

Thus, the results in (20), (21) also apply to the outage probability defined via the total interference power. This complements the results in [3] obtained in terms of the characteristic function with compact, closed-form expressions

\footnotetext{
${ }^{2}$ which may sometimes be not the largest one (due to the effect of Rayleigh fading). However, as we show below, the nearest interferer contribution dominates the tail of the total interference distribution and thus the outage probability.
}

for the outage probability and also explicitly demonstrates the effect of cancelling $(k-1)$ nearest interferers.

The intuition behind Theorem 3 is that the distribution in (6) is much more heavily-tailed (slowly-decaying) than the Rayleigh distribution so that outage events in the combined distribution are mostly caused by nearby interferers without deep Rayleigh fades and the combined distribution is a slightly shifted version of the original one (without fading).

\section{B. Impact of log-normal and combined fading}

This can be analysed in a similar way. The main results are summarized as follows. When the interferers are subject to the average path loss and log-normal i.i.d. fading, and when $(k-1)$ nearest interferers are cancelled, the outage probability is

$$
\mathcal{P}_{\text {out }} \approx \frac{M_{k m / \nu}}{k !}\left(\frac{\bar{N}_{\max }}{D^{m / \nu}}\right)^{k},
$$

where $M_{k m / \nu}=\exp \left(\frac{1}{2}(\sigma k m / \nu)^{2}\right)$ is $k m / \nu$-th moment of the log-normal random variable,

$$
M_{k m / \nu}=\frac{1}{\sqrt{2 \pi} \sigma} \int_{0}^{\infty} x^{k m / \nu-1} \exp \left(-\frac{(\ln x)^{2}}{2 \sigma^{2}}\right) d x,
$$

and $\sigma$ is the standard deviation. The case when no interferers are cancelled corresponds to $k=1$. Comparing (24) to (10), we conclude that the effect of log-normal fading is a shift by a constant factor $>1$, i.e. strictly negative as opposed to Rayleigh fading where it can be either positive or negative. The beneficial effect of cancelling $(k-1)$ nearest interferers is also offset by fading in this case (since $M_{k m / \nu}$ increases with $k$ ). Since the regular varying (heavy tail) nature of the distribution is preserved, Theorem 3 also holds in this case, i.e. the nearest interferer is still dominant.

Likewise, one can consider the combined effect of Rayleigh and log-normal fading. The outage probability is

$$
\mathcal{P}_{\text {out }} \approx \frac{\Gamma(k m / \nu+1) M_{k m / \nu}}{k !}\left(\frac{\bar{N}_{\max }}{D^{m / \nu}}\right)^{k},
$$

and Theorem 3 also applies. Note that the effects of Rayleigh and log-normal fading are multiplicative in terms of the shift constant, and the heavy tail of the distribution, which is due to the Poisson spatial distribution of the interferers and the average path loss, is not affected.

\section{The impact of a broad class of fading distributions}

The results above are not limited to Rayleigh or log-normal fading but rather hold for a broad class of distributions whose tails are dominated by the tail of $P_{a k}$.

Theorem 4: Let the interferers be subject to the average path loss and fading, $P_{i}=g_{i} P_{a i}$, where $g_{i}$ is the fading power gain, i.i.d. for each interferer, and the fading distribution tail is dominated by that in (10), i.e. ,

$$
\lim _{x \rightarrow \infty} \operatorname{Pr}\left(g_{i}>x\right) x^{k m / \nu}=0,
$$

then the outage probability is

$$
\mathcal{P}_{\text {out }} \approx \frac{M_{k m / \nu}}{k !}\left(\frac{\bar{N}_{\text {max }}}{D^{m / \nu}}\right)^{k}, \text { for large } D,
$$


where $M_{k m / \nu}$ is $k m / \nu$-th moment of the fading power gain, $M_{k m / \nu}=\int_{0}^{\infty} x^{k m / \nu} f_{g}(x) d x$, and $f_{g}(x)$ is the pdf of $g$. Furthermore, the nearest interferer dominates the outage events, i.e. Theorem 3 holds, and, thus, the outage in (28) holds in terms of both the total and nearest interferer's power.

Proof: along the same lines as that of Theorem 3 and that of (20).

It should be noted that Theorem 4 includes almost all popular fading models, i.e. Rayleigh, Rice, Nakagami, Weibul, log-normal, or any distribution whose tail decays faster than polynomially. It is also interesting to note that the fading enters the outage probability only via the moment $M_{k m / \nu}$ and the condition of tail dominance, all other details being irrelevant. The effect of fading is positive for $M_{k m / \nu}<1$ and negative for $M_{k m / \nu}>1$.

Finally, the effect of fading can also be considered jointly with partial interference cancellation, and the outage probabilities in (15), (16) are respectively modified to

$$
\begin{gathered}
\mathcal{P}_{\text {out }} \approx \alpha^{m / \nu} M_{m / \nu} \bar{N}_{\max } D^{-m / \nu} \\
\mathcal{P}_{\text {out }} \approx \frac{\alpha^{(k-1) m / \nu} M_{(k-1) m / \nu}}{(k-1) !}\left(\frac{\bar{N}_{\text {max }}}{D^{m / \nu}}\right)^{k-1}
\end{gathered}
$$

i.e. the multiplicative constant shift of the outage probability is preserved. The required partial cancellation levels in (17) and (18) are modified to

$$
\begin{gathered}
\alpha<\frac{1}{D^{1 /(k-1)}}\left(\frac{M_{k m / \nu} \bar{N}_{\max }}{M_{(k-1) m / \nu} \cdot k}\right)^{\nu / m(k-1)} \\
\alpha<\frac{1}{D^{k-1}}\left(\frac{M_{k m / \nu} \bar{N}_{\max }^{k-1}}{M_{m / \nu} \cdot k !}\right)^{\nu / m}
\end{gathered}
$$

Noting that $M_{k m / \nu}$ increases with $k$ for Rayleigh, log-normal and composite fading, its effect on the required cancellation level is beneficial in both cases (i.e. higher $\alpha$ is acceptable), and it is more pronounced for the case of partial cancellation of $(k-1)$ nearest interferers. This is intuitively explained by the fact that these fading distributions decay very fast (exponentially or sub-exponentially) at the large signal region but only polynomially at the low signal region and, thus, the fading results more often in a weaker signal than in a stronger one.

\section{CONCLUSION}

A model of interference in wireless networks with Poisson spatial distribution of the nodes is considered. The nearest interferer power, which dominates the total one, is used to define the outage probability. This simplifies the analysis significantly, results in compact, closed-form characterisation of the outage probability, including the case where some interferers are cancelled, either completely or partially, and allows to compare different cancellation strategies and to find the required level of cancellation. The effect of fading is characterized for a broad class of distributions, including all popular fading models and in combination with the effect of interference cancellation. Our main findings in terms of the node density - outage probability tradeoff at the low outage region can be summarized as follows:

- when $(k-1)$ strongest interferers are cancelled completely or near completely (see (17), (18)), the upper bound scales as $\mathcal{P}_{\text {out }}^{1 / k}$, i.e. much higher node density can be tolerated for larger $k$ (see (12));

- when strongest interferers are partially cancelled by the level independent of the INR, the upper bound still scales as $\mathcal{P}_{\text {out }}$, with a fixed improvement due to interference cancellation (see (15), (16));

- with linear filtering, the upper bound scales as $\mathcal{P}_{\text {out }}$, with a fixed improvement due to the filtering (see [11] for details);

- when fading is present, the scaling above still holds (with an additional fixed multiplicative constant, which depends on fading distribution - see (28)-(30)).

Thus, the main conclusion here is that complete or near complete cancellation of strongest interferers is essential to go from $\mathcal{P}_{\text {out }}$ to $\mathcal{P}_{\text {out }}^{1 / k}$ scaling.

\section{REFERENCES}

[1] E.S. Sousa, J.A. Silvester, Optimum Transmission Ranges in a DirectSequence Spread-Spectrum Multihop Packet Radio Network, IEEE JSAC, v. 8, N. 5, pp. 762-771, Jun. 1990.

[2] E.S. Sousa, Performance of a Spread Spectrum Packet Radio Network Link in a Poisson Field of Interferers, IEEE Trans. Information Theory, v. 38, N. 6, pp. 1743-1754, Nov. 1992.

[3] J. Ilow, D. Hatzinakos, Analytic Alpha-Stable Noise Modeling in a Poisson Field of Interferers or Scatterers, IEEE Trans. Signal Processing, v. 46, N. 6, pp. 1601-1611, Jun. 1998.

[4] J. Ilow, D. Hatzinakos, A. Venetsanopoulos, Performance of FH SS Radio Networks with Interference Modeled as a Mixture of Gaussian and AlphaStable Noise, IEEE Trans. Communications, v. 46, N. 4, pp. 509-520, Apr. 1998.

[5] S. P. Weber et al, Transmission Capacity of Wireless Ad Hoc Networks With Outage Constraints, IEEE Trans. Information Theory, v. 51, N. 12, pp. 4091-4102, Dec. 2005.

[6] S. P. Weber et al, Transmission Capacity of Wireless Ad Hoc Networks With Successive Interference Cancellation, IEEE Trans. Information Theory, v. 53, N. 8, pp. 2799-2814, Aug. 2007.

[7] S. P. Weber et al, The Effect of Fading, Channel Inversion, and Threshold Scheduling on Ad Hoc Networks, IEEE Trans. Information Theory, v. 53, N. 11, pp. 4127-4149, Nov. 2007.

[8] G. L. Stuber, Principles of Mobile Communication (2nd Ed.), Kluwer, Boston, 2001.

[9] V.I.Mordachev, "Statistical Characteristics of Dynamic Range of Inadvertent Disturbances with Space-Scattered Groupings of Their Sources", in Proc. of the 9-th Wroclaw Symp. on EMC, June 1988, pp.571-576.

[10] V.Mordachev, Mathematical Models for Radiosignals Dynamic Range Prediction in Space-Scattered Mobile Radiocommunication Networks, IEEE VTC Fall, Boston, Sept. 24-28, 2000.

[11] V.I. Mordachev, S. Loyka, Node Density - Outage Probability Tradeoff in Wireless Networks, 2008 IEEE International Symposium on Information Theory, Toronto, Canada, July 2008.

[12] G. Samorodnitsky, M.S. Taqqu, Stable Non-Gaussian Random Processes, Chapman\&Hall/CRC, Boca Raton, 1994.

[13] M. Haenggi, On Distances in Uniformly Random Networks, IEEE Trans. Information Theory, v. 51, N. 10, pp. 3584-3586, Oct. 2005. 